\title{
Mystery Case: Anti-NMDAR encephalitis with overlapping demyelinating syndrome
}

Hyun Woo Kim, MD, Christopher Lamb, MD, Sheheryar Jamali, MD, and

Alfonso Sebastian Lopez Chiriboga, MD

Neurology ${ }^{\circledR}$ 2020;94:e1866-e1869. doi:10.1212/WNL.0000000000009320

\section{Pearls}

- Anti-NMDA receptor encephalitis can coexist with an overlapping demyelinating syndrome.

- An atypical presentation of a single autoimmune disorder should prompt investigation for coexistent autoimmune disorders.

- Discovery of overlap syndromes is important because the management and prognosis may be different.

\section{Oysters}

- In autoimmune encephalitis, shorter time from symptom onset to treatment initiation has been associated with better outcome. ${ }^{1}$

- $\quad$ Treatment should not be delayed until the result of autoantibody testing is available.

\section{Case report}

A 31-year-old man developed a subacute onset of headache, left-sided numbness, and anterograde amnesia. In the following 2 weeks, he experienced personality changes, anxiety, paranoid thoughts, $7 \mathrm{~kg}$ weight loss, and worsening cognitive changes. He said that he felt as though he was trapped in a time loop, meaning that events seemed to be constantly recurring to him. He denied fever, night sweats, viral prodromal symptoms, or recent vaccinations. He was diagnosed with acute disseminated encephalomyelitis (ADEM) in 2007 from which he made a full recovery. On neurologic examination, he was inattentive and agitated. His word registration and short-term recall at 5 minutes were 0 of 5 . He had verbal and motor perseveration with echolalia. He had decreased sensation to pinprick on his entire left side. He also had left pronator drift and bilateral athetosis of the upper limbs. The remainder of his examination was normal. His overall clinical examination finding was consistent with a multifocal process involving subcortical and cortical regions.

On day 1, routine blood testing including complete blood cell count with differential, metabolic panel, liver function test, RPR, ESR, ANA, thyroid function tests, thiamine, B12, urinalysis, urine toxicology screen, heavy metal screening, and HIV were normal or negative. Brain MRI revealed multiple T2 hyperintensities within the right internal capsule, periventricular white matter, and bilateral mesial temporal lobes. There were contrast-enhancing lesions involving the right dorsal pons and right internal capsule and thalamus (figure, A-F). CSF analysis demonstrated 9 nucleated cells $/ \mu \mathrm{L}$ with lymphocytic predominance. On day $2, \mathrm{CT}$ of the chest, abdomen, and pelvis and testicular ultrasound were negative for malignancy. Cervical and thoracic spine MRI was performed to look for any past or active demyelinating lesions, especially in the setting of previous history of ADEM, and the result was normal. Video EEG did not demonstrate abnormal ictal or interictal epileptiform discharges. By this time, CSF Gram stain, cultures, and viral PCRs returned negative, and 8 CSF-specific oligoclonal bands were present. Serum and CSF NMDA-R IgG and serum MOG-IgG1 antibodies were sent to the laboratory. On hospital day 3, IV methylprednisolone

\author{
Correspondence \\ Dr. Kim \\ Kim.hyun1@mayo.edu
}

\section{MORE ONLINE}

Survey and results

NPub.org/mc9417 
Figure Brain MRI findings in anti-NMDAR encephalitis with overlapping MOG-IgG-associated demyelinating syndrome (A) Axial T2-FLAIR brain MRI from September 2018 showing right greater than left T2 hyperintensities in the mesial temporal lobes, (B) in the right posterior limb of the internal capsule, and (C) in the right periventricular white matter tracts. (D) Axial T1 gadolinium-enhanced brain MRI showing right pontine crescent-shaped contrast enhancement and (E) punctate enhancement in the right pons and (F) in the right posterior limb of the internal capsule and thalamus. (G and H) Axial T2-FLAIR brain MRI from June 2007 showing T2 hyperintensities in the right subcortical region and periventricular white matter tracts. (I and J) Axial T2-FLAIR brain MRI from November 2007 showing largely resolved lesions.

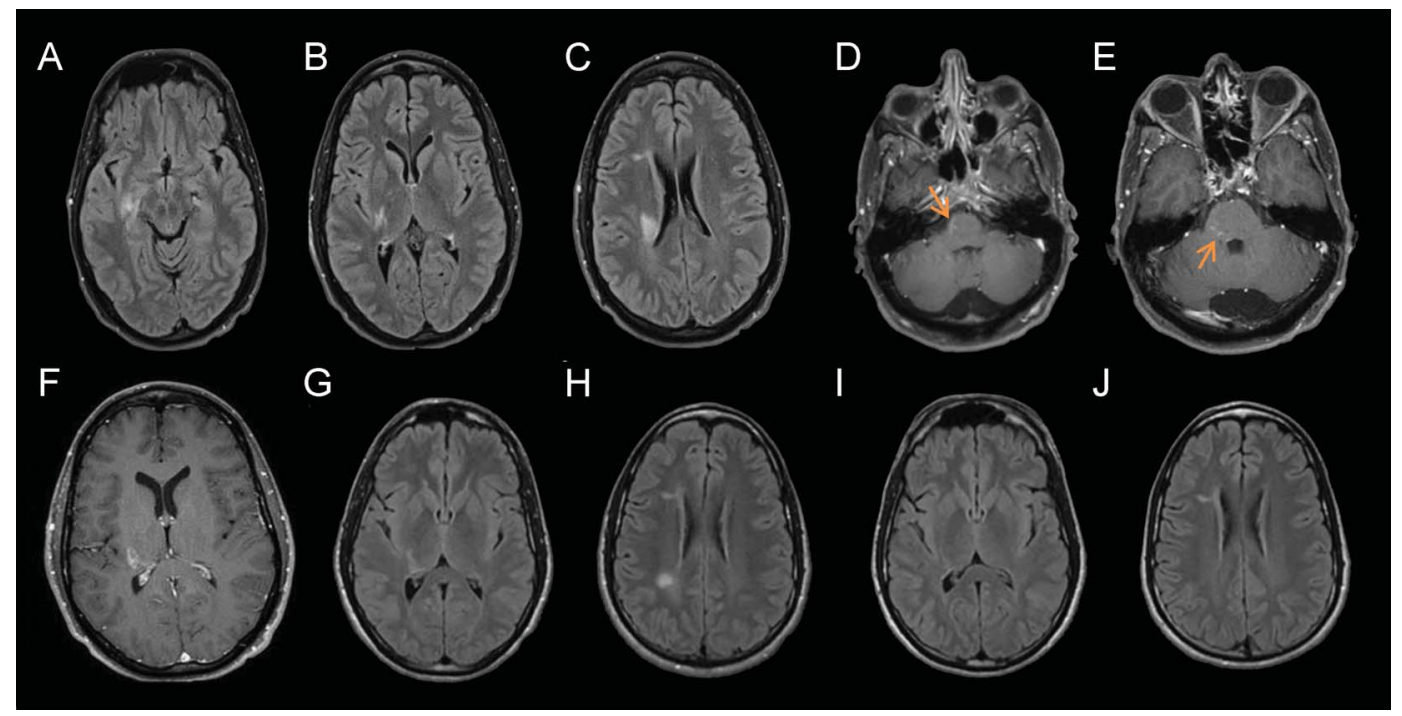

$1000 \mathrm{mg}$ was started and repeated daily for 5 total days. Left hemihypesthesia and pronator drift improved after treatment, and immediate follow-up brain MRI revealed resolution of contrast enhancement. However, his cognitive changes and neuropsychiatric symptoms persisted. His Montreal Cognitive Assessment (MOCA) score was 10 of 30 despite steroid treatment. Hence, on day 8 , he was started on 5 rounds of plasmapheresis (PLEX). After PLEX, he became much calmer, his time loop ended, and his MOCA score improved to 19 of 30. His CSF NMDA-R IgG antibody and serum MOG-IgG1 returned positive. He was diagnosed with anti-NMDAR encephalitis with overlapping MOG-IgG-associated demyelinating syndrome (MOGAD). He was started on longterm maintenance therapy with rituximab.

On 6-month follow-up, his MOCA score improved to 25 of 30 with resolution of neuropsychiatric symptoms and memory issues. He is planning to go back to work.

\section{Discussion}

We present a rare case of subacute autoimmune encephalitis with clinical features of both anti-NMDAR encephalitis and MOG-associated disorder (MOGAD), which was not fully encompassed by either entity alone. Anti-NMDAR encephalitis with overlapping demyelinating syndrome is rare, but has previously been described in the literature. In a large retrospective analysis of 691 patients with anti-NMDAR encephalitis, ${ }^{2} 11$ of 691 patients had anti-NMDAR encephalitis occurring simultaneously with additional clinical and MRI features inconsistent with anti-NMDAR encephalitis. Only 2 patients were seropositive for MOG-IgG1 antibodies with features that would be atypical for anti-NMDAR encephalitis alone, such as ataxia, unilateral hemiparesis, or infratentorial MRI abnormalities.

Our patient presented with clinical features that are typical of anti-NMDAR encephalitis: headache, behavioral changes, abnormal movements (athetosis, chorea, and dystonia), memory loss, and speech disturbance. He did not develop other symptoms, such as autonomic dysregulation or severe encephalopathy requiring intensive care unit admission, which was perhaps related to the prompt recognition of the diagnosis and early treatment with immunotherapy. ${ }^{3}$ Brain MRI can be normal in as many as $50 \%$ of patients with antiNMDAR encephalitis, although multiple abnormalities have been described in some patients including nonspecific T2 hyperintensities, most commonly in mesial temporal lobes, but also in the cerebellar or cerebral cortex, subcortical regions, or brainstem. ${ }^{4}$ In our patient, brain MRI demonstrated heterogeneous T2 hyperintensities in the bilateral mesial temporal lobes (figure, A). These lesions did not resemble demyelinating lesions, as there was an absence of confluent T2 hyperintensity or contrast enhancement.

Our patient's presentation also included several features atypical of NMDAR encephalitis. His left hemihypesthesia and pronator drift are better explained by overlapping demyelinating syndrome because unilateral sensorimotor deficits are rarely seen in anti-NMDAR encephalitis. His sensorimotor symptoms were linked to MRI findings of demyelination including confluent T2 hyperintensity of the right thalamus, 
internal and external capsule, peripheral diffusion restriction, and rim enhancement along the leading edge of inflammation in $\mathrm{T} 1$ postgadolinium sequence ${ }^{5}$ (figure, $\mathrm{C}-\mathrm{F}$ ). These atypical features led to additional testing for MOG-IgG1 in serum. He did meet the criteria for the diagnosis of coexisting MOGAD with previous history of ADEM, MOG-IgG seropositivity, and MRI findings compatible with CNS demyelination. ${ }^{6}$ Our patient did not have other clinicoradiographic features of MOGAD, such as longitudinally extensive spinal cord lesions, conus medullaris lesions, or perioptic gadolinium enhancement of the optic nerves. ${ }^{7}$ MOGAD is generally highly responsive to corticoseroids, ${ }^{6}$ and his unilateral sensorimotor symptoms resolved rapidly on finishing 5 doses of IV methylprednisolone, whereas neuropsychiatric symptoms resulting from antiNMDAR encephalitis took several weeks to resolve.

The exact mechanism and frequency of overlapping antiNMDAR and demyelination is uncertain. His previous presentation of encephalitis with diagnosis of ADEM occurred in 2007, a time when MOG-IgG antibody testing was not commercially available. A brain MRI obtained in 2007 at the time of his ADEM diagnosis revealed lesions in the right periventricular white matter tracts, which fully resolved months after the initial onset of symptoms (figure, G-J). Our patient's recurrent episode in 2018 affected these areas again (figure, B and C). Recurrence of disease in the same location has been described in patients with relapsingremitting ADEM. ${ }^{8}$ Therefore, his case likely represents relapsing MOGAD, and it is quite possible that the patient was seropositive in 2007, as persistent seropositivity is associated with an increased risk of relapses. ${ }^{9}$

Although our patient has coexistent MOG and NMDAR antibodies, the initial treatment remains similar to that of a patient with either antibody alone. First-line immunotherapy consists of corticosteroids, IVIG, PLEX, or a combination of the 3 . In patients with anti-NMDAR encephalitis associated with malignancy, tumor excision should also be performed. In patients whose symptoms are refractory to first-line therapy, additional second-line immunotherapy, such as rituximab or cyclophosphamide, is needed. ${ }^{3}$ If relapse occurs, a long-term maintenance immunotherapy after rescue treatment may reduce the likelihood of future relapses. Although initial treatment approach for anti-NMDAR encephalitis and demyelinating disorders is similar, subsequent management strategies for recurrence of symptoms, prognosis, and risk of recurrence could be different, which highlights the importance in diagnosing both conditions. ${ }^{2}$ Surveillance for relapse is more challenging because neurologist, patient, and family members need to monitor for relapses of both disorders, including not only typical neuropsychiatric symptoms of NMDAR encephalitis but also heterogeneous manifestations associated with MOGAD. Also, as in our case, subsequent treatment plan was more complex because his residual neuropsychiatric symptoms and executive dysfunction had to be managed with multidisciplinary approach including psychiatry and comprehensive rehabilitation.
In summary, NMDAR encephalitis can occur simultaneously with MOGAD. If a patient presents with aforementioned demyelinating features that are atypical for NMDAR encephalitis alone, serum MOG-IgG1 should be tested. Likewise, clinicians should have a low threshold to test for other neural autoantibodies in patients who have a diagnosis of MOGAD who present with atypical features such as movement disorders or neuropsychiatric symptoms.

\section{Study funding}

No targeted funding reported.

\section{Disclosure}

The authors report no disclosures relevant to the manuscript. Go to Neurology.org/N for full disclosures.

\section{Appendix Authors}

\begin{tabular}{lll}
\hline Name & Location & Contribution \\
\hline $\begin{array}{l}\text { Hyun Woo Kim, } \\
\text { MD }\end{array}$ & $\begin{array}{l}\text { Mayo Clinic, } \\
\text { Jacksonville, Florida, } \\
\text { Florida }\end{array}$ & $\begin{array}{l}\text { Manuscript concept } \\
\text { and design, drafting of } \\
\text { the manuscript, editing of } \\
\text { the text, and review of the } \\
\text { literature }\end{array}$ \\
\hline $\begin{array}{l}\text { Christopher } \\
\text { Lamb, MD }\end{array}$ & $\begin{array}{l}\text { Mayo Clinic, } \\
\text { Jacksonville, Florida, }\end{array}$ & $\begin{array}{l}\text { Patient care, major } \\
\text { contribution to the } \\
\text { editing of the text, and } \\
\text { revision for intellectual } \\
\text { content }\end{array}$ \\
\hline $\begin{array}{l}\text { Sheheryar } \\
\text { Jamali, MD }\end{array}$ & $\begin{array}{ll}\text { Mayo Clinic, } \\
\text { Jacksonville, Florida, } \\
\text { Florida }\end{array}$ & $\begin{array}{l}\text { Contributed to manuscript } \\
\text { editing }\end{array}$ \\
\hline $\begin{array}{l}\text { Alfonso Lopez } \\
\text { Chiriboga, MD }\end{array}$ & $\begin{array}{l}\text { Mayo Clinic, } \\
\text { Jacksonville, Florida, } \\
\text { Florida }\end{array}$ & $\begin{array}{l}\text { Critical review of the } \\
\text { manuscript and revision } \\
\text { for intellectual content } \\
\text { and major contribution } \\
\text { to the editing of } \\
\text { the text }\end{array}$ \\
& &
\end{tabular}

\section{References}

1. Lancaster E. The diagnosis and treatment of autoimmune encephalitis. J Clin Neurol 2016;12:1-13.

2. Titulaer MJ, Höftberger R, Iizuka T, et al. Overlapping demyelinating syndromes and anti-N-methyl-D-aspartate receptor encephalitis. Ann Neurol 2014;75:411-428.

3. Dalmau J, Lancaster E, Martinez-Hernandez E, Rosenfeld MR, Balice-Gordon R Clinical experience and laboratory investigations in patients with anti-NMDAR encephalitis. Lancet Neurol 2011;10:63-74.

4. Dalmau J, Geis C, Graus F. Autoantibodies to synaptic receptors and neuronal cell surface proteins in autoimmune diseases of the central nervous system. Physiol Rev 2017;97:839-887.

5. Tillema JM, Pirko I. Neuroradiological evaluation of demyelinating disease. Ther Adv Neurol Disord 2013;6:249-268.

6. Jarius S, Paul F, Aktas O, et al. MOG encephalomyelitis: international recommendations on diagnosis and antibody testing. J Neuroinflammation 2018;15:134.

7. Jarius S, Ruprecht K, Kleiter I, et al. MOG-IgG in NMO and related disorders: a multicenter study of 50 patients. Part 2: epidemiology, clinical presentation, radiological and laboratory features, treatment responses, and long-term outcome. J Neuroinflammation 2016;13:280.

8. Cohen O, Steiner-Birmanns B, Biran I, Abramsky O, Honigman S, Steiner I. Recurrence of acute disseminated encephalomyelitis at the previously affected brain site. Arch Neurol 2001;58:797-801.

9. López-Chiriboga AS, Majed M, Fryer J, et al. Association of MOG-IgG serostatus with relapse after acute disseminated encephalomyelitis and proposed diagnostic criteria for MOG-IgG-associated disorders. JAMA Neurol 2018;75:1355-1363. 


\section{Mystery Case responses: A 31-year-old with left-sided numbness, amnesia, and personality changes}

The Mystery Case series was initiated by the Neurology ${ }^{\circledR}$ Resident \& Fellow Section to develop the clinical reasoning skills of trainees. Residency programs, medical student preceptors, and individuals were invited to use this Mystery Case as an educational tool. Responses to multiple-choice questions formulated using this case were solicited through a group email sent to the American Academy of Neurology Consortium of Neurology Residents and Fellows and through social media. We received 580 responses. The majority of respondents (66\%) had just been in practice for $1-4$ years; $56 \%$ were residents or fellows, whereas $34 \%$ were faculty/board-certified physicians; the remainder were medical students or advanced practice providers. Seventy percent resided outside the United States. A wide range of practice settings was represented.

When presented with this brief vignette and the brain MRI of a 31-year-old with left-sided numbness, amnesia, and personality changes and asked for the 2 most likely differential diagnoses, $58.3 \%$ correctly chose autoimmune encephalitis, and $37.6 \%$ correctly recognized demyelinating lesions. The most frequently selected incorrect options were CNS lymphoma (32.6\%), tertiary syphilis (15.3\%), and MELAS (14.3\%), these are lower in the differential.

After being given preliminary results, the participants were asked for the 3 most important next tests. Autoimmune encephalitis panel was correctly chosen by $70.7 \%$. Because of the suspicion of demyelination, the other correct answers included whole-spine MRI with contrast (28.1\%) and AQP4 and MOG antibodies (37.1\%). The most commonly selected incorrect answers were CSF cytology and flow cytometry (46.4\%), CSG HSV PCR (21.0\%), and syphilis treponemal test (16.7\%). Although these tests are reasonable, they are lower in priority for this patient.

Finally, the participants were asked for the best empiric treatment while waiting for results. IV methylprednisolone was correctly selected by $55.9 \%$. The most common incorrect answers were IV acyclovir (17.9\%) and no therapeutic intervention (14.0\%). This patient testing came back with a positive anti-NMDAR antibody and anti-MOG antibody. He was diagnosed with anti-NMDAR encephalitis with overlapping MOG-IgG-associated demyelinating syndrome. He received IV steroids, followed by PLEX with good response. This overlap syndrome has been previously described, and in a study, $5 \%$ of patients with anti-NMDAR encephalitis showed evidence of demyelinating disease. ${ }^{1}$ Antibodies against AQP4 and MOG have been reported in these cases. ${ }^{1,2}$ Although acute treatment is similar, recognition of these association is important to guide long-term treatment.

Alonso Zea Vera, MD

Cincinnati Children's Hospital Medical Center

\section{References}

1. Titulaer MJ, Hoftberger R, lizuka T, et al. Overlapping demyelinating syndromes and anti-N-methyl-D-aspartate receptor encephalitis. Ann Neurol 2014;75:411-428.

2. Hacohen $Y$, Absoud M, Hemingway C, et al. NMDA receptor antibodies associated with distinct white matter syndromes. Neurol Neuroimmunol Neuroinflamm 2014;1:e2. 


\section{Neurology}

Mystery Case: Anti-NMDAR encephalitis with overlapping demyelinating syndrome Hyun Woo Kim, Christopher Lamb, Sheheryar Jamali, et al.

Neurology 2020;94;e1866-e1869 Published Online before print March 31, 2020

DOI 10.1212/WNL.0000000000009320

This information is current as of March 31, 2020

Updated Information \&

Services

References

Subspecialty Collections

Permissions \& Licensing

Reprints including high resolution figures, can be found at: http://n.neurology.org/content/94/17/e1866.full

This article cites 11 articles, 1 of which you can access for free at: http://n.neurology.org/content/94/17/e1866.full\#ref-list-1

This article, along with others on similar topics, appears in the following collection(s):

Acute disseminated encephalomyelitis

http://n.neurology.org/cgi/collection/acute_disseminated_encephalomy elitis

All Clinical Neurology

http://n.neurology.org/cgi/collection/all_clinical_neurology

Autoimmune diseases

http://n.neurology.org/cgi/collection/autoimmune_diseases

Information about reproducing this article in parts (figures,tables) or in its entirety can be found online at:

http://www.neurology.org/about/about_the_journal\#permissions

Information about ordering reprints can be found online:

http://n.neurology.org/subscribers/advertise

Neurology ${ }^{\circledR}$ is the official journal of the American Academy of Neurology. Published continuously since 1951, it is now a weekly with 48 issues per year. Copyright @ 2020 American Academy of Neurology. All rights reserved. Print ISSN: 0028-3878. Online ISSN: 1526-632X.

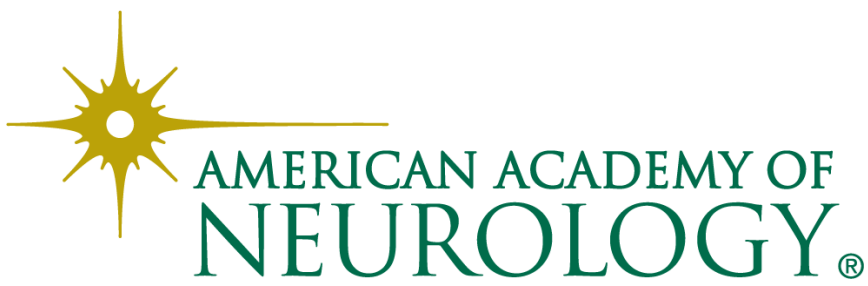

